\title{
Isolating the non-polar contributions to the intermolecular potential for water-alkane interactions
}

\author{
Deepti Ballal, Pradeep Venkataraman, Wael A. Fouad, Kenneth R. Cox, \\ and Walter G. Chapman ${ }^{\text {a) }}$ \\ Department of Chemical and Biomolecular Engineering, Rice University, 6100 S. Main, Houston, \\ Texas 77005, USA
}

(Received 17 April 2014; accepted 23 July 2014; published online 12 August 2014)

\begin{abstract}
Intermolecular potential models for water and alkanes describe pure component properties fairly well, but fail to reproduce properties of water-alkane mixtures. Understanding interactions between water and non-polar molecules like alkanes is important not only for the hydrocarbon industry but has implications to biological processes as well. Although non-polar solutes in water have been widely studied, much less work has focused on water in non-polar solvents. In this study we calculate the solubility of water in different alkanes (methane to dodecane) at ambient conditions where the water content in alkanes is very low so that the non-polar water-alkane interactions determine solubility. Only the alkane-rich phase is simulated since the fugacity of water in the water rich phase is calculated from an accurate equation of state. Using the SPC/E model for water and TraPPE model for alkanes along with Lorentz-Berthelot mixing rules for the cross parameters produces a water solubility that is an order of magnitude lower than the experimental value. It is found that an effective water Lennard-Jones energy $\varepsilon_{W} / k=220 \mathrm{~K}$ is required to match the experimental water solubility in TraPPE alkanes. This number is much higher than used in most simulation water models (SPC/E$\varepsilon_{W} / k=78.2 \mathrm{~K}$ ). It is surprising that the interaction energy obtained here is also higher than the water-alkane interaction energy predicted by studies on solubility of alkanes in water. The reason for this high water-alkane interaction energy is not completely understood. Some factors that might contribute to the large interaction energy, such as polarizability of alkanes, octupole moment of methane, and clustering of water at low concentrations in alkanes, are examined. It is found that, though important, these factors do not completely explain the anomalously strong attraction between alkanes and water observed experimentally. (C) 2014 AIP Publishing LLC. [http://dx.doi.org/10.1063/1.4892341]
\end{abstract}

\section{INTRODUCTION}

Models of solubility of water in hydrocarbons are important in hydrocarbon production and processing to prevent condensation leading to corrosion and flow assurance issues related to gas hydrate formation. Understanding the interactions between water and non-polar molecules has implications in biological applications as well, where hydrophobic effects have been widely studied. ${ }^{1,2}$ While most studies look at the solubility of hydrocarbons in water, here we study the solubility of water in hydrocarbons, particularly in n-alkanes. While this system requires a relatively simple intermolecular potential model that is not computationally intensive, the results provide a rigorous test of the water-alkane interaction potential.

Although interactions between molecules involve manybody effects, molecular simulation models generally approximate the potential as effective two-body interactions. Alkanes are approximated in most models ${ }^{3,4}$ as chains of segments that interact via a spherically symmetric Lennard-Jones (LJ) potential,

$$
u_{L J}(r)=4 \varepsilon\left[\left(\frac{\sigma}{r}\right)^{12}-\left(\frac{\sigma}{r}\right)^{6}\right]
$$

\footnotetext{
a) Author to whom correspondence should be addressed. Electronic mail: wgchap@ rice.edu. Tel.: (1) 713348 4900. Fax: (1) 7133485478.
}

where $\sigma$ is the $\mathrm{LJ}$ diameter, $\varepsilon$ is the $\mathrm{LJ}$ energy, and $\mathrm{r}$ is the distance between the centers of two segments. Water is usually modeled as a rigid non-polarizable molecule with a spherically symmetric Lennard-Jones potential and off-center partial charges that interact via a coulombic potential. Most popular water models are similar in the form of the intermolecular potential but differ in their parameters, such as LJ energy, number of charged sites, and distance and angle between the sites. ${ }^{5-9}$

These simple potential models have been fairly successful in describing the pure component properties of water and alkane but fail to reproduce the properties of a mixture of water and alkane. ${ }^{10}$ Various quantum and classical studies have concentrated on better understanding water-alkane interactions. ${ }^{11-16}$ In classical models, the water-alkane cross interaction parameters $\left(\sigma_{A W}\right.$ and $\left.\varepsilon_{A W}\right)$ are obtained from pure component parameters using the Lorentz-Berthelot rule

$$
\sigma_{A W}=\frac{\sigma_{A}+\sigma_{W}}{2}, \quad \varepsilon_{A W}=\xi \sqrt{\varepsilon_{A} \varepsilon_{W}},
$$

where $\sigma_{A}$ and $\sigma_{W}$ are the $\mathrm{LJ}$ diameters for alkane and water, respectively; $\varepsilon_{A}$ and $\varepsilon_{W}$ are LJ energy parameters for alkane and water, respectively; and $\xi$ is an empirical parameter that corrects for errors in the Berthelot rule. Based on the first term in the London dispersion equation, we expect $\xi<1.0$. 
Most studies agree that the water-alkane interaction energy obtained as a geometric mean of water and alkane energies does not describe the properties of the mixture, but there is still disagreement on what the interaction energy should be. Paschek, ${ }^{13}$ who studied the solubility of methane in a waterrich phase, concluded that the low solubility of simulated methane in water compared to experiments can be attributed to two factors: (1) poor prediction of density of water at given conditions by most models and (2) ignoring the polarizability of methane. Docherty et al. ${ }^{14}$ used a TIP4P/2005 model for water, which is known to produce accurate densities of water, and found a qualitative improvement in the dependence of the solubility of methane on temperature but still could not get quantitative agreement. They had to use a correction of 1.07 to the Berthelot rule $\left(\varepsilon_{M W}=1.07 \sqrt{\varepsilon_{M} \varepsilon_{W}} ; \varepsilon_{M W} / k=117.3 \mathrm{~K}\right)$ to match the experimental solubility which they argued could be justified based on the polarizability of methane being ignored in the study. Dyer et al. ${ }^{12}$ included the polarizability of methane explicitly into the model, but still could not successfully match the experimental solubility leaving the question of the ability of these potential models to reproduce properties of the mixture unanswered. Ashbaugh et al. ${ }^{17,18}$ recognizing this problem, reparameterized the TraPPE model for alkanes to match alkane hydration free energies in a TIP4P/2005 water model. Interestingly, in the theoretical work of Pratt and Chandler, ${ }^{19}$ a methane-water interaction energy of $\varepsilon_{M W} / k$ $=168.5 \mathrm{~K}$ was required to match the Henry's Law constant of methane in water. This is significant because they used the experimentally obtained structure for the pure water phase and not a model predicted structure. To obtain this water-methane interaction energy with SPC/E water and TraPPE methane would require a $\xi=1.56$.

While the above studies have looked at the limit where methane is present in low concentration in water, a few studies have looked at water in a hydrocarbon rich phase and the energies suggested by these studies are very different. Johansson et al. ${ }^{20}$ looked at the solubility of water in decane (instead of methane) and found that a correction factor of 1.3 was required to the Berthelot rule $\left(\varepsilon_{A W}=1.3 \sqrt{\varepsilon_{A} \varepsilon_{W}} ; \varepsilon_{A W} / k=139.9 \mathrm{~K}\right)$ to match the solubility of water in decane. van Buuren et al. ${ }^{10}$ while modeling a water-decane liquid-liquid interface, could not describe both the bulk water properties and water-decane interfacial properties with a single value of water energy. They had to use different energies for water interacting with itself and for water interacting with alkane $\left(\varepsilon_{W} / k=78.2 \mathrm{~K}\right.$ for water-water interaction and $\varepsilon_{W} / k=277.8 \mathrm{~K}$ for water-alkane interaction) to be able to simultaneously describe the bulk and interfacial properties. These examples point towards an inconsistency in the parameters being used in the potential model for wateralkane interactions.

Since alkanes primarily interact via non-polar interactions and water has strong polar interactions, the dominant interactions in the alkane-rich region and water-rich region are different. All the previous studies have looked at systems that have significant hydrogen bonding between the water molecules. It would be inaccurate to evaluate the non-polar interactions in a system where polar interactions are dominant. In the limit of very low water content in alkanes, there are neg- ligible water-water interactions and water interacts only with the non-polar alkanes. The important interactions here are the alkane-alkane and alkane-water non-polar interactions. In this case one can investigate how the non-polar interactions affect the properties of the system independent of the charges in the water model. The non-polar parameters are important not only for solubility of water in alkanes but also affect the interfacial properties of alkanes and water such as surface tension and the sharpness of the interface. ${ }^{10}$

The purpose of this study is to isolate the effective water/alkane non-polar pair potential by matching the solubility of water in the alkane phase using molecular simulation for the alkane rich phase and an accurate correlation for the water rich phase. A transferable potential model is demonstrated for the water in alkane system. Section II describes the methodology used to evaluate the water-alkane interaction in an alkane-rich phase. Section III describes the results from the solubility studies and discusses contributions from polarizability, distributed charges on the alkane, and water clustering.

\section{METHODOLOGY}

The solubility of water in different alkanes (pentane to dodecane) was calculated from Monte Carlo simulation using the towhee simulation package ${ }^{21}$ with alkanes modeled using the TraPPE model ${ }^{3}$ and water with the SPC/E model. ${ }^{5}$ Since the solubility of water in the alkane-rich phase is very low at ambient conditions, Henry's Law is a good approximation for the fugacity of water in this phase. The Henry's Law constant for water in alkane was calculated using Widom test particle insertion. ${ }^{22}$ The mole fraction of water in the alkane-rich phase $\left(x_{\text {water }}\right)$ is obtained from the fugacity of water $\left(f_{\text {water }}\right)$ in the water-rich phase and Henry's Law constant $(H)$ for water in the alkane rich phase

$$
x_{\text {water }}=\frac{f_{\text {water }}}{H} .
$$

The solubility of alkane in water is negligible at the conditions of interest, so the fugacity of water in the water rich phase is approximated by the fugacity of pure water. Instead of using the fugacity of water from the SPC/E model, which is known to be inaccurate, the water fugacity was obtained from an accurate equation of state by Saul and Wagner. ${ }^{23,24}$ The Lorentz-Berthelot rule was used to obtain the cross interaction parameters. All calculations were carried out in a canonical ensemble (NVT) with 256 alkane molecules at $298 \mathrm{~K}$. The density of alkanes at $298 \mathrm{~K}$ and $1 \mathrm{~atm}$ was obtained from the NIST database. ${ }^{25}$ To ensure that there was no system size dependence, Widom test particle insertion of water in boxes of different sizes was performed using GROMACS. ${ }^{26}$ Insertion of water in a box containing 10000 and 50000 methane molecules gave the same results as insertion in a box containing 256 methane molecules.

\section{RESULTS AND DISCUSSION}

The solubility of water in n-alkanes at $298 \mathrm{~K}$ and 1 atm obtained from simulation is compared with the experimental data in Figure 1. Using the SPC/E model for water and 


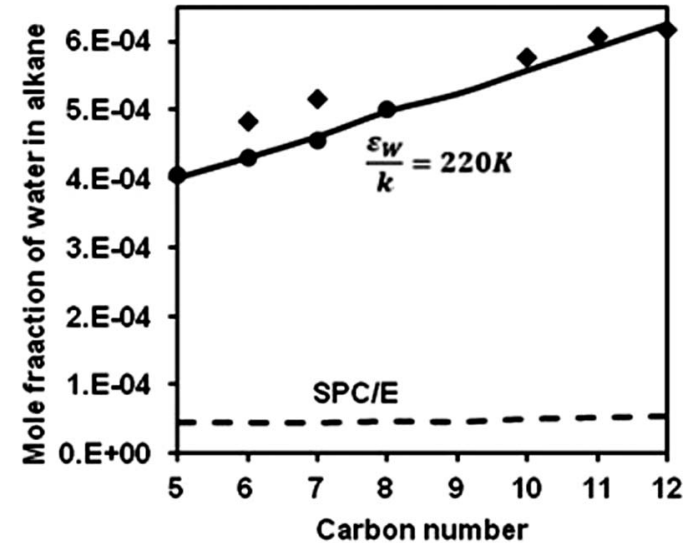

FIG. 1. Solubility of water in different alkanes with varying carbon number at $298 \mathrm{~K}$ and $1 \mathrm{~atm}$. Symbols are experimental data, ${ }^{27,28}$ solid line is obtained using parameters obtained here and the dashed line is obtained using the SPC/E model of water.

TraPPE model for n-alkanes, the calculated solubility of water is found to be an order of magnitude lower than the experimental values. ${ }^{27,28}$ We show the results for only the SPC/E model of water here, but most popular models of water would give similar results because they use similar LJ parameters $\left(\varepsilon_{W} / k=78.197 \mathrm{~K}\right.$ and $\left.\sigma_{W}=3.16 \AA\right)$. We recognize that interactions with higher multipole moments on alkanes are not being considered, but these are typically neglected in current simulation models. This assumption will be discussed in detail later.

The case of water content in n-alkanes provides the opportunity to fit the LJ interactions between water and nalkanes independently of the charged sites and the simulated water structure. We have fit the LJ parameters for water to match the experimental solubility in the alkanes. Since water interacts only with alkanes via non-polar interactions, water can be looked at as a Lennard-Jones sphere in alkanes. Widom test particle insertions of these LJ spheres in TraPPE alkanes require water $\mathrm{LJ}$ parameters of $\varepsilon_{W} / k=220 \mathrm{~K}$ and $\sigma_{W}$ $=3.0 \AA\left(\varepsilon_{W-\mathrm{CH}_{3}} / k=147 \mathrm{~K}\right.$ and $\left.\varepsilon_{W-\mathrm{CH}_{2}} / k=100.5 \mathrm{~K}\right)$ to match the experimental water solubility. The LorentzBerthelot (LB) mixing rule is assumed while obtaining the water parameters here. Though the LB rule may not be very accurate, the water-alkane interaction energy here is too high ( $\xi=1.67)$ to be attributed to the inadequacy of the LB rule. Values of LJ parameters close to the ones we find here were obtained in the theoretical work of Emborsky et al. ${ }^{29}$ when they calculated the solubility of water in alkanes using the PC-SAFT model for alkanes. They found that a value of $\varepsilon_{W} / k=207 \mathrm{~K}$ was required to match the solubility of water in the different alkanes.

The dependence of solubility on the LJ diameter was also considered. The size of the water molecule was varied within the range that is generally considered acceptable from our experimental knowledge of water systems $(2.8-3.2 \AA)$ and the difference in solubility was found to be within $10 \%$. This is very different from the water-rich phase where it has been noted that the van der Waals radius affects the properties of the system significantly. ${ }^{10,30}$ But here we find that in the

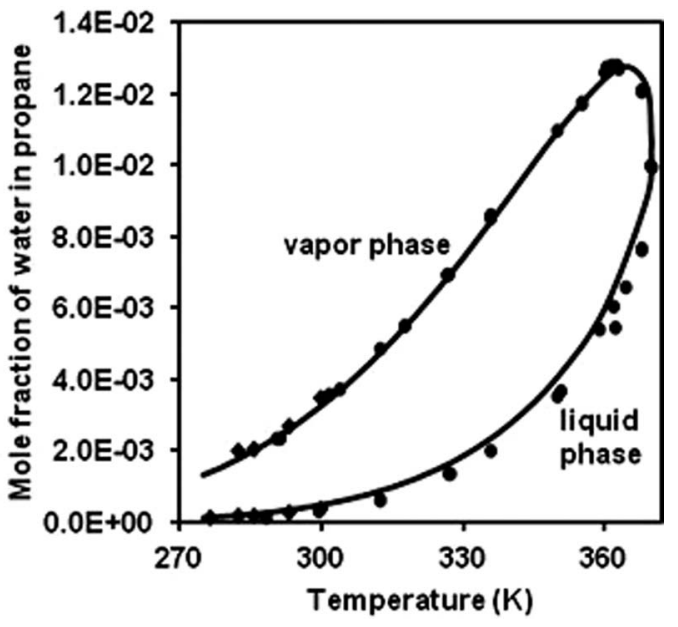

FIG. 2. Partitioning of water between liquid and vapor phases of propane. Symbols are experimental $\mathrm{data}^{31,32}$ and the line is simulation prediction using an energy $\varepsilon_{W} / k=220 \mathrm{~K}$.

alkane-rich phase, the water solubility is more sensitive to the interaction energy than the size of the molecules.

Figure 2 shows the partitioning of water between coexistent liquid and vapor phases of propane. Densities for liquid and vapor propane were obtained from NIST database ${ }^{25}$ and separate simulations were done to calculate the chemical potential for insertion of water in the liquid and vapor phase. Using the energy and diameter of water obtained from insertions of a LJ sphere representative of water $\left(\varepsilon_{W} / k=220 \mathrm{~K}\right.$ and $\sigma_{W}$ $=3.0 \AA$ ) in alkanes gives good agreement with experimental values $^{31,32}$ over a range of temperatures even though the energy and diameter were fit to data at $298 \mathrm{~K}$. There is some error in the solubility of water in the liquid phase at high temperatures but the experimental data could be matched by changing the energy for water by less than $10 \%\left(\varepsilon_{W} / k\right.$ $=200 \mathrm{~K})$. Figure 3 shows the solubility of water in methane at various temperatures and pressures compared to experimental data. ${ }^{23}$ Agreement with the data is very good over

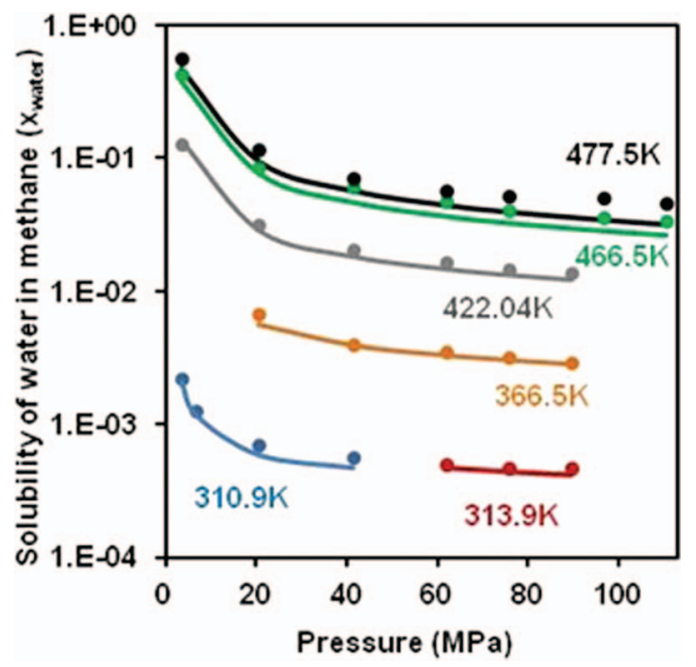

FIG. 3. Solubility of water in methane at different temperature and pressures. Symbols are experimental data ${ }^{23}$ and lines are simulation results using a water energy of $\varepsilon_{W} / k=220 \mathrm{~K}$. 
a wide range of temperature and pressure. The simulations underpredict the solubility at higher concentrations because there are appreciable water-water interactions in that region and Henry's Law would not apply. With water energy of 220 $\mathrm{K}$, the water-methane interaction energy is $\varepsilon_{M W} / k=180.5 \mathrm{~K}$ which means a correction to the Berthelot rule of 1.65 when using the SPC/E model for water and the TraPPE model for methane. The correction here is much higher than what was suggested previously by studies of methane solubility in water ${ }^{14,33}$ with one exception. The proposed value is only 7\% higher than the interaction energy proposed by Pratt and Chandler. ${ }^{19}$

Interestingly, high interaction energy between water and non-polar molecules is not restricted to alkanes alone. A large value for water-argon interaction energy was also found by Bickes et al. ${ }^{34}$ when they fit a Lennard-Jones potential to their results from molecular beam scattering experiments for the water-argon systems. They found a water-argon interaction energy of $164 \mathrm{~K}$; using the energy for argon to be $118 \mathrm{~K}^{35}$ gives a water energy parameter of about $228 \mathrm{~K}$. Harvey ${ }^{36}$ used this water-argon energy to calculate the second virial coefficient and found reasonable agreement with experimental values. These studies, using mixtures of water with different nonpolar molecules, all point towards a different intermolecular potential between water and non-polar molecules than what is used in most simulation models today.

We will now analyze the reasons why the water-alkane interaction is more attractive than expected. One such reason is the polarizability. The ability of water molecules to polarize alkane molecules (dipole-induced dipole interaction) in a water-rich phase is different than in an alkane-rich phase due to the different dielectric medium. This will affect the water-alkane interactions differently in the two regions. Since including polarizability in the simulation is computationally intensive, polarizability is typically neglected and a nonpolarizable model is used instead. It has been claimed that the larger than expected attraction between water and alkanes is due to neglecting the dipole-induced dipole interaction. Solubility studies of non-polar solutes in water explicitly accounting for polarizability have been inconclusive. ${ }^{12}$ It has also been suggested that the polarization correction included in the non-polarizable SPC/E model creates an "overpolarized" environment creating an artificially large dislike for non-polar environments decreasing the solubility of water in non-polar solvents. ${ }^{37}$

To study the effect of polarizability and other contributions we will focus on the solubility of water in methane. For a water-methane pair in vacuum, the different contributions to the interaction energy can be calculated using molecular properties like polarizability, ionization potential, and dipole moment. ${ }^{38}$ The dipole-induced dipole interaction accounts for about $13 \%$ of the total energy; it might be imagined that a cumulative effect might arise from multiple water-methane pairs available. So we explicitly included polarizability in the model to study a water-methane mixture. Two water molecules (POL3 potential ${ }^{39}$ ) in 5000 methane molecules (polarizable TraPPE potential ${ }^{12}$ ), i.e., $x_{\text {water }}=4$ $\times 10^{-4}$, were simulated in $\operatorname{AMBER}(\mathrm{v} .11)^{40}$ at a temperature of $313.9 \mathrm{~K}$ and pressure of $89.67 \mathrm{MPa}$. We compare the poten-

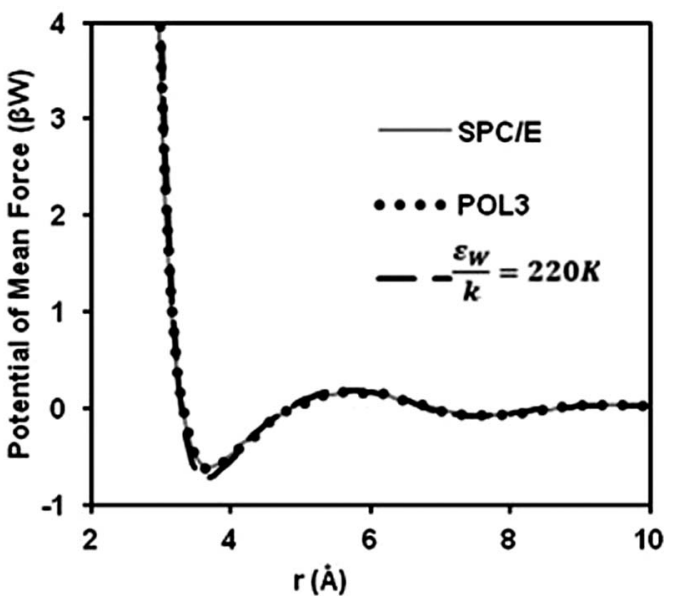

FIG. 4. Water-methane potential of mean force for non-polarizable (SPC/E and LJ water sphere with $\varepsilon_{W} / k=220 \mathrm{~K}$ in TraPPE methane) and polarizable model (POL3 water in polarizable TraPPE methane).

tial of mean force between water and methane with and without polarizability. Figure 4 shows the water-methane potential of mean force for the non-polarizable models (SPC/E and LJ sphere with $\varepsilon_{W} / k=220 \mathrm{~K}$ in TraPPE methane) and polarizable model (POL3 water in polarizable TraPPE methane). The difference in the potential of mean force between water and methane for the polarizable and non-polarizable models is statistically insignificant. The structure of methane around the water is not affected by polarizability and weakly affected by the change in the water-methane interaction energy.

Another assumption made in the study is neglecting the partial charges on methane that are responsible for octupole-octupole interaction between methane molecules and octupole-dipole interactions between water and methane. To test this assumption, we modeled methane molecules with explicit hydrogen atoms using Optimized Potential for Liquid Simulation - All Atom (OPLS-AA) parameters. Widom test particle insertion of water in a system containing 20 water molecules and 50000 methane molecules at $313.9 \mathrm{~K}$ and $89.67 \mathrm{MPa}$ gave the solubility of water as $x_{\text {water }}=1.18$ $\times 10^{-4}$ which is higher than the SPC/E solubility $9.74 \times 10^{-5}$ but still lower than the experimental solubility of $4.67 \times 10^{-4}$.

Finally, the assumption that water molecules do not interact with one another at such low concentrations is tested. If there were significant water-water interactions then the assumption that the insertion of water in a pure alkane system gives the chemical potential of water at the solubility limit would not be valid. The amount of water-water interaction or clustering required for SPC/E water to match the experimental chemical potential was calculated using a method proposed by Tripathi and Chapman. ${ }^{41}$ The method gives the chemical potential of inserting a water molecule in a system at the solubility limit using the chemical potential of insertion of a water "monomer" $\left(\mu_{\text {mono }}\right)$ and the fraction of these water monomers $\left(X_{\text {mono }}\right)$. The excess chemical potential is given by

$$
\mu_{w a t e r}^{e x}=k T \ln \left(\frac{X_{m o n o}}{\exp (-\beta \Delta u)_{N}}\right)
$$


where $\Delta u$ is the insertion energy for the monomer. The experimental chemical potential of inserting water into methane at $313.9 \mathrm{~K}$ and $89.63 \mathrm{MPa}$ is $-1.44 \mathrm{~kJ} / \mathrm{mol}$ whereas that for the insertion of SPC/E water in TraPPE alkanes is $2.78 \mathrm{~kJ} / \mathrm{mol}$. These simulations and test particle insertions were performed using GROMACS molecular dynamics package. ${ }^{26}$ Using Eq. (4), to obtain the experimental chemical potential, around $80 \%$ of water molecules would have to be in clusters $\left(X_{\text {mono }}\right.$ $=0.2$ ) which is much higher than the amount of clustering we observed in our simulations of water molecules at the experimental solubility limit $(22 \%)$ and what is reported in the literature. ${ }^{20,42}$ The amount of clustering is the same for SPC/E water in TraPPE methane and OPLS-AA methane. This analysis leads us to believe that even though polarizability, clustering, and octupole moment of methane are important, they do not completely explain the high water-alkane interaction energy required to match the solubility of water in an alkanerich phase.

\section{CONCLUSIONS}

It is clear that the interaction between water and nonpolar molecules is not being modeled correctly by conventional intermolecular potential models. Here we studied the water-alkane interaction in the limit of low water content where non-polar interactions could be evaluated independent of the polar components of the potential. A larger than expected water-alkane energy was required to match the solubility of water in alkane; much larger than obtained in the limit of low alkane solubility in water, the other end of the concentration regime. The solubility of water in alkanes was much more sensitive to the water-alkane interaction energy than the diameter of water which is not the case for solubility of alkanes in water where the diameter is very important. Certain assumptions made such as neglecting the polarizability of alkane, the octupole moment of methane, and clustering of water were tested. Though neglecting these factors contributes to the errors in solubility using conventional models, these factors do not explain the effective interaction between water and alkane necessary to match the experimental solubility data. The research community lacks a complete picture of water-alkane interactions at the molecular level. As discussed here, studies of water/alkane interactions must consider water in nonpolar solvents in addition to the commonly studied nonpolar solutes in water.

\section{ACKNOWLEDGMENTS}

We thank Thi Vo and John W. Chapman for early simulation studies of water solubility in methane. We also thank Carlos Vega, Ilja Siepmann, Allan Harvey, Ariel Chialvo, and Dilip Asthagiri for helpful discussions. We gratefully acknowledge the financial support from the Robert A. Welch Foundation (Grant No. C-1241) and of RPSEA (Grant No. 1021-4204-01). D.B. gratefully acknowledges ConocoPhillips for financial support of a Ph.D. fellowship. W.A.F. gratefully acknowledges the Abu Dhabi National Oil Company (ADNOC) for financial support through a Ph.D. scholarship.
${ }^{1}$ A. Cooper, Biophys. Chem. 115(2-3), 89-97 (2005).

${ }^{2}$ J. C. Rasaiah, S. Garde, and G. Hummer, Annu. Rev. Phys. Chem. 59, $713-$ 740 (2008).

${ }^{3}$ M. G. Martin and J. I. Siepmann, J. Phys. Chem. B 102(14), 2569-2577 (1998)

${ }^{4}$ W. L. Jorgensen, D. S. Maxwell, and J. Tirado-Rives, J. Am. Chem. Soc. 118(45), 11225-11236 (1996).

${ }^{5}$ H. Berendsen, J. Grigera, and T. Straatsma, J. Phys. Chem. 91(24), 62696271 (1987)

${ }^{6}$ W. L. Jorgensen, J. Chandrasekhar, J. D. Madura, R. W. Impey, and M. L. Klein, J. Chem. Phys. 79, 926 (1983).

${ }^{7}$ W. L. Jorgensen and J. D. Madura, Mol. Phys. 56(6), 1381-1392 (1985).

${ }^{8}$ M. W. Mahoney and W. L. Jorgensen, J. Chem. Phys. 112, 8910 (2000).

${ }^{9}$ J. L. Abascal and C. Vega, J. Chem. Phys. 123, 234505 (2005).

${ }^{10}$ A. R. van Buuren, S. J. Marrink, and H. J. C. Berendsen, J. Phys. Chem. 97(36), 9206-9212 (1993).

${ }^{11}$ G. Bolis, E. Clementi, D. Wertz, H. Scheraga, and C. Tosi, J. Am. Chem. Soc. 105(3), 355-360 (1983).

${ }^{12}$ P. J. Dyer, H. Docherty, and P. T. Cummings, J. Chem. Phys. 129, 024508 (2008).

${ }^{13}$ D. Paschek, J. Chem. Phys. 120, 6674 (2004).

${ }^{14}$ H. Docherty, A. Galindo, C. Vega, and E. Sanz, J. Chem. Phys. 125, 074510 (2006).

${ }^{15}$ B. J. Anderson, J. W. Tester, and B. L. Trout, J. Phys. Chem. B 108(48), 18705-18715 (2004).

${ }^{16}$ O. Akin-Ojo and K. Szalewicz, J. Chem. Phys. 123(13), 134311 (2005).

${ }^{17}$ H. S. Ashbaugh, N. J. Collett, H. W. Hatch, and J. A. Staton, J. Chem. Phys. 132, 124504 (2010).

${ }^{18}$ H. S. Ashbaugh, L. Liu, and L. N. Surampudi, J. Chem. Phys. 135, 054510 (2011).

${ }^{19}$ L. R. Pratt and D. Chandler, J. Chem. Phys. 67(8), 3683-3704 (1977).

${ }^{20}$ E. Johansson, K. Bolton, D. N. Theodorou, and P. Ahlström, J. Chem. Phys. 126, 224902 (2007).

${ }^{21}$ See http://towhee.sourceforge.net for MCCCS Towhee simulation package.

${ }^{22}$ B. Widom, J. Phys. Chem. 86(6), 869-872 (1982).

${ }^{23} \mathrm{~A}$. Saul and W. Wagner, "International equations for the saturation properties of ordinary water substance," J. Phys. Chem. Ref. Data 16(4), 893-901 (1987).

${ }^{24}$ M. Yarrison, K. R. Cox, and W. G. Chapman, Ind. Eng. Chem. Res. 45(20), 6770-6777 (2006)

${ }^{25}$ E. W. Lemmon, M. O. McLinden and D. G. Friend, "Thermophysical Properties of Fluid Systems" in NIST Chemistry WebBook, NIST Standard Reference Database Number 69, Eds. P. J. Linstrom and W. G. Mallard, National Institute of Standards and Technology, Gaithersburg MD, 20899, http://webbook.nist.gov.

${ }^{26}$ B. Hess, C. Kutzner, D. Van Der Spoel, and E. Lindahl, J. Chem. Theory Comput. 4(3), 435-447 (2008).

${ }^{27}$ J. Polak and B. C. Y. Lu, Can. J. Chem. 51(24), 4018-4023 (1973).

${ }^{28}$ C. Tsonopoulos, Fluid Phase Equilib. 156(1), 21-33 (1999).

${ }^{29}$ C. P. Emborsky, K. R. Cox, and W. G. Chapman, Ind. Eng. Chem. Res. 50(13), 7791-7799 (2011).

${ }^{30}$ B. Chen, Ph.D. thesis, University of Minnesota, 2001.

${ }^{31}$ K. Y. Song and R. Kobayashi, Fluid Phase Equilib. 95, 281-298 (1994).

${ }^{32}$ R. Kobayashi and D. Katz, Ind. Eng. Chem. 45(2), 440-446 (1953).

${ }^{33}$ O. Konrad and T. Lankau, J. Phys. Chem. B 109(49), 23596-23604 (2005).

${ }^{34}$ R. Bickes, Jr., G. Duquette, C. Van den Meijdenberg, A. Rulis, G. Scoles, and K. Smith, J. Phys. B: At. Mol. Phys. 8(18), 3034 (1975).

${ }^{35}$ A. Sherwood and J. Prausnitz, J. Chem. Phys. 41, 429 (1964).

${ }^{36}$ P. H. Huang and A. H. Harvey, in TEMPMEKO 2004, Faculty of Mechanical Engg. and Naval Arch., Zagreb, Vol. 1, pp. 351-356.

${ }^{37}$ L. Vlcek, A. A. Chialvo, and D. R. Cole, J. Phys. Chem. B 115(27), 87758784 (2011).

${ }^{38}$ J. N. Israelachvili, Intermolecular and Surface Forces, Revised 3rd ed. (Academic Press, 2011).

${ }^{39}$ J. W. Caldwell and P. A. Kollman, J. Phys. Chem. 99(16), 6208-6219 (1995).

${ }^{40}$ D. A. Case, T. E. Cheatham, T. Darden, H. Gohlke, R. Luo, K. M. Merz, A. Onufriev, C. Simmerling, B. Wang, and R. J. Woods, J. Comput. Chem. 26(16), 1668-1688 (2005).

${ }^{41}$ S. Tripathi and W. G. Chapman, Mol. Phys. 101(8), 1199-1209 (2003).

${ }^{42}$ K. Bolton, E. Johansson, L. Jönsson, and P. Ahlström, Mol. Simul. 35(1011), 888-896 (2009). 